\title{
Residual Limb Complications and Management Strategies
}

\author{
Blaise A. Pascale $\cdot$ Benjamin K. Potter
}

Published online: 17 September 2014

(C) Springer Science+Business Media New York (Outside the USA) 2014

\begin{abstract}
Major extremity amputations due to any cause are fraught with potential and overt complications, ranging from infection and heterotopic ossification to phantom neuropathic pain and symptomatic neuromata. Prevention of issues such as bursa formation and skin irritation or ulceration through intimate, comfortable prosthetic socket fit, and diligent skin care are crucial and vastly preferred to managing the sequelae of same. For most issues other than deep infection, a trial of non-operative modalities is indicated prior to any operative intervention, as conservative management is often successful. For patients with persistently symptomatic residual limbs and an identifiable cause, focal or complete operative revision can be highly successful at alleviating symptoms and improving patient prosthetic tolerance and function. A multi-disciplinary approach from an integrated rehabilitation team is critical to the successful management of these challenging and rewarding patients.
\end{abstract}

Keywords Amputation · Complication · Infection, heterotopic ossification $\cdot$ Neuroma $\cdot$ Myodesis $\cdot$ Bursitis

Investigation performed at the Uniformed Services University of Health Sciences and Walter Reed National Military Medical Center, Bethesda, Maryland.

B. A. Pascale · B. K. Potter

Department of Surgery, Uniformed Services University of

Health Sciences, Bethesda, MD, USA

B. K. Potter ( $\square)$

Department of Orthopaedics and Rehabilitation, Walter Reed

National Military Medical Center, 8901 Wisconsin Ave,

America Building (19), 2nd Floor - Ortho, Bethesda, MD 20889,

USA

e-mail: Benjamin.k.potter.mil@health.mil

\section{Introduction}

The definition of health is the state of complete physical, mental, and social well being and not merely the absence of disease or infirmity [1]. Persons with limb loss-amputees-have sustained a severe insult to their physical, mental, and social health. The care of these patients is a complicated, important matter. Physical medicine principles aim to restore function to amputees following surgery, as despite an apparently successful surgery, patients will generally not utilize a prosthesis that is painful [2], and thus may not be restored to their optimal functional potential. The post-operative course of amputees, especially those due to trauma and diabetes or peripheral vascular disease, can be plagued by complications potentially leading to surgical revision [3]. The medical team is responsible for collaborating and overcoming these complications to achieve optimal physical and psychosocial functional recovery.

This article addresses residual limb complications and their management strategies. First, we discuss surgical principles, pertinent history and physical findings, and pain types experienced by amputees. Next, we discuss the presentation and management of soft tissue, osseous, and dermatologic complications associated with residual limbs following major extremity amputations.

\section{General Surgical Principles}

In order to produce a robust, healthy, and painless functional residual limb, surgical goals during and following amputation surgery include length preservation with hemorrhage control, thorough debridement of wounds or nonviable tissue, and removal of any malignant tissue with 
wide margins for tumor-related amputations. Excision of any devitalized tissue without soft tissue attachments and simple, voluminous bulb syringe, or gravity flow irrigation intraoperatively helps to decrease bacterial counts in traumatic or infected wounds [4]. Utilization of the remaining viable muscle and other fasciocutaneous tissue allows for generation of a durable and well-padded limb [4]. Healthy tissue with adequate padding for the distal bone end using intraoperative myodesis, myoplasty, and/or myofascial techniques has been shown by biomechanical studies to assist with better control and alignment of the terminal residual limb [5, 6]. Classically, surgeons are trained to evaluate for final closure based on the " $4 \mathrm{C}$ 's" - color, consistency, contraction, and circulation [7]. Traumatic amputations should be left open for serial debridement procedures, while primary amputations-those outside the zone of injury, infection, vascular compromise, or tumorcan often be closed at the time of surgery.

The standard of care for combat-related amputations, in order to provide optimum healing potential, is the interval use of negative pressure wound therapy between debridement procedures. This therapy mitigates the risk of contamination and tissue necrosis while encouraging vascularity [8] and has largely supplanted historic, conventional skin traction for distal coverage facilitation. Therapy with antibiotic-impregnated polymethylmethacralate (PMMA) beads has been shown to be effective in contaminated animal models [9]. In a 2011 review, Barth et al. [10 ] analyzed several studies that resulted in infection control rates of $80-100 \%$ when antibiotic beads were used in conjunction with systemic antibiotics; while encouraging these results were from non-controlled and non-randomized studies. Despite this lack of definitive evidence of efficacy, PMMA beads are often used as a local adjuvant to decrease the bacterial load of the soft tissue envelope [4].

After a technically sound surgery, prevention of residual limb complications starts with proper post-operative wound care. Early residual limb complications result in setbacks that can substantively retard rehabilitation progress. Moreover, sites of early skin breakdown, even after healing, may never achieve the durability of uncompromised, native tissue-an ounce of prevention is, truly, worth a pound of cure. Soft dressings, which we prefer given our high volume of traumatic and trauma-related amputations, allow for easier wound inspection, earlier mobilization, and decreased risk of pressure ulceration; rigid dressings are sometimes preferred to minimize edema, avoid contracture formation, and improve pain control [11, 12]. Dressing philosophies frequently differ between providers and institutions, but as long as they are used appropriately, no method has been convincingly demonstrated to produce superior outcomes [4]. As soon as all tubes and drains are removed, we recommend transitioning to an elastic shrinker stocking over a light dressing to prepare the limb for a prosthesis by reducing edema and molding residual limb contour and shape. Early physical therapy helps to prevent contracture formation and deconditioning and likely has psychological benefits by encouraging use of the residual limb and preventing feelings of dependence and hopelessness.

\section{History, Physical, and Residual Limb Assessment}

A systematic approach to evaluate the residual limb sets both the patient and provider up for success, as potential problem areas are less likely to be missed. While the obvious inclination is to focus largely on the residual limb, completing a full, detailed history remains critical. The provider should obtain key clinical data including the history of original injury or disease, amputation level(s) and technique, patient demographic information, medical comorbidities, and any infections or revision operative procedures. Other key parts of the history include recent trauma, recent change in function or symptoms, along with age and adjustment frequency of prosthesis, daily prosthetic usage, recreational activities, functional goals, and other physical or psychosocial barriers to achieving them.

The history should be followed by a general systemic and musculoskeletal exam evaluating for stability, strength, contractures, and other organic residual limb complications [13]. Direct observation of prosthesis application and removal, standing, and ambulation are standard, as discomfort from inadequate fit, improper shape of margins, and skin irritation or breakdown represent frequent contributors to patient discomfort [14]. More serious anatomic complications, such as potential myodesis failure, or occult soft tissue deficiencies, can be analyzed using plain and weight-bearing radiographs; video fluoroscopy or formal gait analysis may be useful for evaluating gait abnormalities. Radiographs of the entire residual limb can screen for any osseous complications-notably arthritis, fracture, heterotopic ossification (HO), or bone spurs-and should comprise part of the initial assessment. Magnetic resonance imaging (MRI) may be employed to detect bursitis and inflammation/infection in the soft tissue or bone marrow, as well as screening for neuroma locations and myodesis patency [13].

\section{Amputation and Amputee Pain}

Post-amputation pain is the most common presenting residual limb issue and amputee complaint. Reviews of posttraumatic lower-limb amputees, when compared with age-matched controls in health surveys, demonstrate that 
amputees often have decreased physical and mental health scores $[15,16]$. Even highly functional and active amputees frequently perceive physical limitations and experience pain. A complete history, as described above, can elucidate the type of pain experienced. The three most common presenting pain scenarios experienced by amputees are generally categorized as phantom sensations, phantom limb pain, or residual limb pain.

Phantom sensations are generally described as itching, pins and needles, or tingling. These are typically not overwhelming, occur in 53-100\% of amputations, and tend to dissipate or stabilize within the first year following amputations [17]. Phantom sensations differ starkly from phantom limb pain, which is defined as nociceptive afferent pain perceived from the amputated/absent portion of the limb [18]. Often described as burning or throbbing, phantom limb pain ranges from mild to intolerable, and is reported to occur in upto $80 \%$ of patients [13]. Conversely, while both phantom sensations and phantom limb pain reside under the umbrella of perceived pain from the central neural axis, residual limb pain (i.e., stump pain) is organic discomfort localized to the residual limb itself. Soft tissue complications, osseous growth, and neuroma formation are the typical culprits of the latter, contributing to this generally chronic, and nagging discomfort, reported in $50-76 \%$ of amputees $[13,17]$.

\section{Soft Tissue Complications}

\section{Edema}

Edema occurs in the progression from surgery to prosthesis use. Shrinkers or compressive bandages are applied after the amputation procedure to mitigate and rapidly improve early edema. Use of these modalities helps to shape the limb after surgery for prosthesis wear. Residual limbs are continually at risk for developing edema. By avoiding a dependent stump position and using shrinker stockings, edema after or between prosthesis wear is often avoided. Persistent late edema may be indicative of prosthesis irritation, bursa formation, or latent infection.

\section{Infection}

Infection of residual limbs is an unfortunate but expected complication that occurs in $20-41 \%$ of amputations due to trauma or vascular disease, especially in one-stage surgery [19•], with much lower rates for tumor-related and pediatric amputations [19•, 20]. Surgeons should counsel patients preemptively about infection being a frequent issue during the reconstructive process, in order to manage patient expectations and mitigate psychological setbacks should an infection develop. This is extremely important because infection often results in additional operative procedures and delays or extends the period of initial rehabilitation.

An erythematous, swollen, and painful limb is the typical presentation for an infection. Classification of the residual limb infections dictates treatment. Early infections occur less than 6 weeks from closure, likely because of preexisting bacterial colonization of the limb at closure. Most are acute, present with a consequent febrile history, and require shorter durations of therapy than late infections. Late infections are typically chronic, result from a latent dormant process or seeding, and can be associated with a draining sinus [13]. Operative debridement of the wound is often required to determine acuity and extent. Another critical discriminator for treatment is whether an infection is superficial (e.g., cellulitis, folliculitis, or focal subcutaneous abscess) versus deep (i.e., involving the deep soft tissues or bone), as the latter virtually always requires operative debridement.

A post-operative hematoma may act as a focus for infection by increasing tension on the wound and weakening the closure, which disrupts blood supply. Tissue necrosis and wound dehiscence are possible outcomes [21]; thus larger, symptomatic hematomas may require operative debridement. However, fluid collections are ubiquitous in amputations during the early post-operative period and, in the absence of overt clinical signs of infection or hematoma-related symptoms, operative debridement simply because a fluid collection exists is unnecessary-most of these fluid collections resolve spontaneously and uneventfully over time [22].

Focal, superficial infections may be managed with prosthetic rest, oral antibiotics, and close follow-up. More diffuse and concerning, but putatively superficial residual limb infection is managed with admission to the hospital, parenteral antibiotics, elevation, and adjacent joint and complete prosthetic rest. Patients should wear their shrinker stocking, and be made non per os after midnight for the first 1 or 2 days in case of the need for operative treatment. Laboratory evaluation should include blood cultures, complete blood count with manual cell differential, and an inflammatory panel. Orthogonal radiographs can identify soft tissue swelling, osseous changes or new interval bone destruction, and HO. More advanced imaging is not typically necessary, but MRI, ultrasound, or indium-11 tagged white blood cell scans may be useful in patients with equivocal findings.

Consultation with an infectious disease specialist is often necessary to best determine type of antibiotics, duration of therapy, and for side-effect monitoring. Early, acute infections typically require shorter durations of antibiotic therapy. Eradication of infection via aggressive 
debridement is the general rule; as noted previously, virtually all deep residual infections require operative debridement. Systemic illness or aggressive infections also necessitate serial irrigation and debridements. After surgery, drains are left in place to monitor the output for 1-3 days. Dressing changes occur daily after post-operative day 2, and if clear of infection, the patients are then transitioned back into a compressive shrinker. Resumption of prosthesis wear and rehabilitation can begin once all evidence of infection has resolved and the residual limb is adequately healed from any related surgeries.

\section{Bursitis}

Bursitis is a common source of frustration for patients, because it can cause symptoms ranging from prosthesisrelated nuisance to disabling pain. De novo formation of adventitious bursae occurs within subcutaneous connective tissue in response to chronic pressure, irritation, and friction [13]. They present as well circumscribed, transilluminant, and fluctuant masses [23]. Deep bursitis between the patient's myoplasty and adjacent terminal bone end can occur as well. Perioperative fluid collections are typical and should be considered hematomas, seromas, or abscesses until proven otherwise, as bursae only develop after the physiological tolerance of involved tissues is exceeded.

Bursae may be either aseptic or septic, and the differential diagnosis should include infection, abscess, and cellulitis. History of symptom duration, constitutional symptoms, or pain with prosthetic wear guides the diagnosis. Complete blood count with manual differential and inflammatory parameters is necessary to rule out infectious processes. Radiography can identify contributory osseous factors. Because of the potential to cause an iatrogenic fistula or septic bursitis, aspiration is only indicated if the clinician suspects an infectious process. Aspiration is never, however, used as a sole management strategy. Any aspirate should undergo gram stain, cell count, and cultures [24]. Septic bursitis or abscess cases require empiric, then culture-specific antibiotic therapy, basing intravenous, or oral antibiotics on severity and clinical status of the patient. Typically, intravenous therapy with transition to oral after clinical response is appropriate. Lack of response to antibiotics necessitates irrigation and debridement; frank abscesses require surgical evacuation.

Prevention is a critical cornerstone of bursitis management. Bursae may develop at areas of inadequate or redundant coverage of bony prominences $[13,23]$. Monitoring for bursa development helps the treatment team identify adaptive and atrophic changes of the residual limb. Ill-fitting prosthetic sockets frequently contribute to bursa formation, so consideration of socket or liner modifications to reduce friction over bursa may prevent bursitis development; consistent excellent socket fit may also prevent bursae from forming altogether. Suspected aseptic bursitis is initially treated with complete prosthetic rest, compression stocking wear, elevation, ice, and NSAID therapy. Resolution of bursitis may be lengthy, but tangible improvement should occur within a few days, otherwise the clinician should re-evaluate for an underlying infectious process. Recurrent or refractory aseptic bursitis may require steroid injection or operative treatment. Because steroid injection can cause atrophy of overlying fat and soft tissue, it is only advocated in patients with a robust soft tissue envelope. Surgical treatment may be appropriate for patients with modifiable organic causes of the bursa, such as bone spurs, $\mathrm{HO}$, large suture knots, or retained implants.

\section{Symptomatic Neuromata}

Deep, proximal traction neurectomies during amputation surgery seek to avoid neuroma-related symptoms, but many amputees still experience the unpleasant presence of a symptomatic neuroma. With literally dozens of proposed treatment or prevention modalities described and none proven consistently effective or superior, neuroma management can be problematic - all transected nerves heal by forming a neuroma; however, not all neuromas become symptomatic. Irritation of the terminal neuroma bulb from normal physiologic stimuli such as pressure (most commonly), stretch, or vascular pulsation can cause symptoms $[4,25]$. The classic presentation of a neuroma is pain over a discreet area with a palpable nodule that yields a positive Tinnel's percussion test, with pain typically radiating proximally along the nerve and/or distally into the phantom limb. Radiographs may reveal osseous issues or retained fragments aggravating the neuroma. MRI can help to localize or confirm the location of a difficult neuroma but is not generally necessary [26]. Symptomatic neuromas may also be detected and treated with injection using high-resolution ultrasound with probe pressure [27•].

Prosthetic socket modification or surgical revision may alleviate a symptomatic neuroma. Diagnostic injection of local anesthetic at the point of maximal tenderness can help verify the diagnosis in recalcitrant cases, and can inform the physician of prognosis if it is eventually excised. Percutaneous neuroma ablation may also be attempted to mitigate symptoms prior to surgical management. If the neuroma is resistant to all conservative measures, early resection is encouraged because of the potential to develop chronic, refractory pain from changes to the central neural axis [28]. Resection of the neuroma may also decrease reliance on neuropathic pain medications [29••]. One promising new modality for neuroma management is targeted muscle reinnervation (TMR). Originally described and developed for improved terminal device control, TMR 
may have the added benefit of decreasing neuroma-related pain and, potentially, phantom pain [30]. Neuroma symptoms are often improved for a majority of amputees following appropriate treatment.

\section{Ulceration}

Pressure ulcers reportedly occur in upto $57 \%$ of residual limbs [31]. Patients with peripheral vascular disease or diabetes mellitus are at increased risk of developing an ulcer, and ulcerations in these patients are also more worrisome and slower to heal [32]. Often starting as a minor abrasion, ulcers result from friction and repetitive pressure generated by poor prosthesis fit or sudden change in activity level, or progress from worsening vascular compromise. Scar location and soft tissue redundancy can also be exacerbating factors sometimes requiring revision, because they contribute to either ulceration or general prosthesis pain despite socket and liner adjustments. Many clinicians opt to continue prosthesis wear with socket and liner adjustments, local wound care, and frequent skin checks; this has been determined to be safe, with one study reporting $64 \%$ of patient ulcers healing in a period of 6 weeks, $25 \%$ reducing in size, and only $9 \%$ having progression or deterioration [33]. Using a vacuum-assisted socket system on limbs with wounds or ulcers was shown to have no significant difference at 6-month follow-up but did allow earlier prosthesis fitting without inhibiting healing [34•*]. If the ulcer fails to heal, treatment strategies include prosthesis rest, advanced local wound care, and sequential additional prosthetic socket and liner adjustments. Truly recalcitrant, progressive, and/or symptomatic ulcerations may require focal soft tissue revision or advanced coverage modalities. Chronic recurring ulcers may eventually develop into a squamous cell carcinoma, or Marjolin's ulcer [13, 35], emphasizing the importance of both prevention and prompt treatment.

\section{Myodesis Failure}

Myodesis is a technique used in amputation surgery to stabilize the bone within the soft tissue of the residual limb. It prevents motion over the bone and provides padding between the bone and prosthesis socket. Securing the muscles with physiologic tension decreases atrophy over time and provides improved function, control, and stability to the residual limb [25]. Although most critical for transfemoral amputations, myodeses are an important aspect of closure for most amputation levels. Myodesis failure is not a common occurrence. In a study reporting reoperative complications in 300 residual limbs, it occurred only in $6 \%$ of patients, many of whom also had other indications for concurrent reoperation [29••].
Though relatively rare, an inadequate or ruptured myodesis can cause debilitating residual limb pain, an inefficient gait cycle, and ulceration. An uncontrolled fall or hyperabduction injury in well-functioning amputees may indicate an acute failure of the myodesis. Physical exam findings depend on the level of amputation, but general findings may include a palpable rent in the area of concern, a new focal area of prominent bone, and/or a change in residual limb control or alignment. Transfemoral amputees may display a flexed and abducted residual limb with bony prominence anterolaterally, weak adduction and extension, and radiographic evidence of excessively flexed and abducted femur within the soft tissue envelope. Transtibial amputees would display a prominent anterior and distal tibia with possible painful snapping sensation when using the residual limb.

Evaluation for myodesis failure should begin by determining the patient's level of function. Repair may not be necessary in low-demand patients. More active patients require a higher level of functioning and may therefore need surgical revision. The provider must balance the duration of non-operative management with the need for revision, as extended conservative treatment can detract from ultimate success because disuse causes scarring and retraction of the muscle, making operative repair more difficult. In some active patients, immediate repair of acutely ruptured myodesis is therefore reasonable.

\section{Osseous Complications}

\section{Bone Mineral Density Loss}

Amputee literature describes decreases of bone mineral density (BMD) in amputated limbs. Studies show significant differences between the amputated and non-amputated limb, most commonly in lower-extremity amputees [3638]. Disuse atrophy is thought to cause BMD decrease, resulting in osteopenia or osteoporosis in residual limbs [37]. Flint et al. found that patients who took longer to bear weight and run in a prosthesis were most likely to experience severe BMD loss, confirming the previous supposition that the disuse atrophy causes BMD loss [39••]. This decrease predisposes residual limbs to fragility fractures from low energy falls or stress fractures with sudden increases in activity level. Among transtibial amputees, residual limb pain has been associated with decreased BMD [37], but this association is not consistent. Rather than BMD loss being a source of pain, the association may be because pain affects the ability to ambulate, and a decrease in ambulation can affect BMD [39••]. Treatment of osteoporosis is typically accomplished using bisphosphonate medications and supplementation with 
Vitamin D (800 IU) and Calcium (1,000 mg). Although effectively reducing incidence of osteoporotic fracture within geriatric populations, it is uncertain whether bisphosphonate management is effective in the younger amputee population and therefore requires additional study.

\section{Bone Spurs}

Bone spurs or osteophytes can be caused by a change in loading pressure of the bone, as in osteoarthritis, or result from periosteal stripping during a surgical procedure or traumatic event, such as amputation. These bony prominences can cause pain due to compression of a neuroma or poor soft tissue coverage and can cause skin ulceration if they are not monitored appropriately. Some procedures and modifications, such as the Ertl tibiofibular synostosis procedure, are used to avoid osteophyte formation, but the general management strategy is to modify the prosthetic socket and minimize pressure over osteophytes. Judicious handling of (and excision of redundant) periosteum at the time of amputation may help prevent osteophyte formation. Surgical excision of the osteophytes is generally successful and may be necessary if conservative treatment does not produce symptomatic relief.

\section{Heterotopic Ossification}

HO is the formation of mature lamellar bone in non-osseous tissue. Lesions are often asymptomatic, but resultant pain or joint stiffness can severely impact patient function. $\mathrm{HO}$ is clinically evident on radiographs at 2 months following initial injury and amputation [20, 40]. Grading for the HO severity may be determined using the Walter Reed system [20], based on anteroposterior and lateral radiographs of the residual limb. Choosing the view that demonstrates the most ectopic bone, the severity is determined based on the cross-sectional area it encompasses. Mild is $<25 \%$ of the area, moderate is $25-50 \%$, and severe is $>50 \%$.

A 2007 retrospective study demonstrated that amputation within the zone of injury, as well as a blast mechanism of injury was predictive of HO. The authors reported an incidence of $63 \%$, but acknowledged selection bias due to poor radiographic follow-up of ostensibly asymptomatic amputees [20]. Even so, the authors noted that if they assumed that those eliminated from the study were free of $\mathrm{HO}$, the incidence would still be $36 \%$, and estimated the actual incidence at just over $50 \%$. A more recent combatrelated amputee analysis revealed a $67 \% \mathrm{HO}$ incidence [29••]. Few analyses of HO incidence are published from civilian centers. Matsumota et al. reports that, contrary to military findings, there is no difference in risk between traumatic and non-traumatic civilian amputees. Rates of

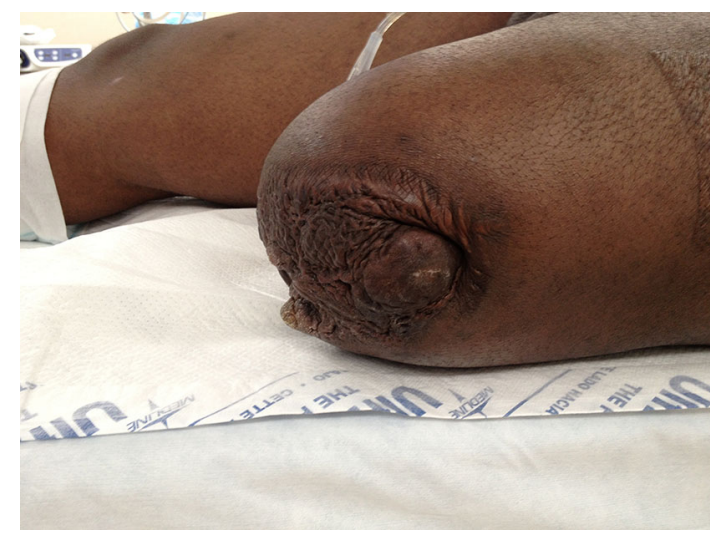

Fig. 1 Preoperative clinical photograph of a left transfemoral amputation sustained as a result of a motorcycle accident. The patient developed symptomatic heterotopic ossification (HO) beneath the terminal split thickness skin graft that caused persistent pain and recurrent ulcerations despite serial prosthetic socket modifications and wound care. He therefore required surgical revision for $\mathrm{HO}$ resection, secondary myodesis revision due to involvement with $\mathrm{HO}$, and concurrent skin graft excision and myofasciocutaneous flap advancement. Focal verrucous hyperplasia is also visible posteriorly

surgical excision of $\mathrm{HO}$ in this civilian population were $11 \%[41 \bullet \cdot$. Military HO excision rates have varied from $18.7 \%$ [20] to $41 \%$ [29*0], with the larger percentage attributed to better follow-up and increased experience with $\mathrm{HO}$ excision leading to more aggressive early excision.

Primary prophylaxis techniques for $\mathrm{HO}$ prevention include a single 5-10 Gy dose of local radiation, NSAID therapy, or etidronate. There are drawbacks to each of these modalities. Radiation may result in wound-related complications and therefore is not recommended in high-energy amputation or patients with other causes of poor wound healing (e.g., peripheral vascular disease or diabetes) [42]. NSAID therapy inhibits fracture healing and is contraindicated in patients with intracranial vascular trauma, and may not be tolerated in some elderly patients with renal compromise or gastritis. Etidronate is FDA-approved for $\mathrm{HO}$ prophylaxis but is actually not effective per a 2004 Cochrane database review [43]. It is also associated with a "rebound" phenomenon when the patient ceases taking it, and delays fracture healing [42]. We therefore do not recommend etidronate for $\mathrm{HO}$ prophylaxis.

If symptomatic, $\mathrm{HO}$ is initially addressed with conservative therapy. Treatment should be individualized for its effect on the patient's daily activities. Surgical excision is typically reserved for pain, ulceration, or joint stiffness specifically attributable to $\mathrm{HO}$ that fails to respond to conservative measures [42]. For many patients, activity modification and tailored socket and liner adjustment can successfully mitigate symptoms and preclude the need for operative excision. When surgical excision is necessary, 
symptom relief is reliably achieved when adequate residual soft tissue coverage exists (Fig. 1). Historically, patients were made to wait for definitive maturation of HO lesions (as indicated by quiescence on bone scans or normalization of serum alkaline phosphatase), but the recent trend is to proceed immediately to surgery after patients fail conservative therapy and symptoms warrant, provided the $\mathrm{HO}$ is well-defined and corticated-typically within 5-6 months of amputation [20, 40].

\section{Dermatologic Complications}

Amputees are continually at risk for dermatologic complications, with studies reporting prevalence rates between 16 and $63 \%$ [31]. The soft tissue flaps typically utilized for residual limb coverage are not physiologically adapted to carry the increased load associated with wearing a prosthesis, so these sites can become irritated. The prosthesis also places the flap tissue in a closed, moist environment ripe for bacterial growth. Additionally, increased use of silicone liners prevents sweat from evaporating or escaping. Increase sweat production associated with wear results in an imbalance of evaporation and production, termed hyperhidrosis [32]. Hyperhidrosis can encourage the development of skin problems in the residual limb. Other provocateurs of skin problems in amputees were identified as the use of antibacterial soap, smoking, and frequency of washing the limb, though the authors were unable to infer a causal relationship from their study of 805 patients. [31].

The most significant factor associated with skin problems is thought to be the level of amputation, with transtibial amputees developing problems four times more frequently than in transfemoral amputees [44]. Transtibial amputations may be more likely to experience complications possibly because they tend to be more active than transfemoral amputees and have an increased number of bony prominences in their residual limbs. Syme's and partial foot amputees were also found to be at an increased risk. In the same study, peripheral vascular disease patients were found to have a decreased risk of skin problems, likely due to lower activity level and prosthesis wear. [44].

\section{Epidermal Hyperplasia}

Proliferation of epidermoid cells in residual limbs can cause a variety of conditions. Verrucous hyperplasia appears as multiple irregular warty papules and plaques as a result of persistent stump edema in unsupported areas of the stump-prosthetic interface [13]. This can be painful and cause skin breakdown, so it is important to equalize the contact pressure throughout the residual limb. Invaginated keratin into the dermis, aggravated by the shear forces of prosthetic wear may result in draining or painful localized masses known as epidermoid cysts [32]. When symptomatic, these cysts should be excised.

\section{Contact Dermatitis}

Allergic contact dermatitis can occur from prosthesis use. Presentation varies from acute swelling and erythema to chronic scaling. The use of a silicone sleeve is thought to prevent sensitization to the component [45], but it is not completely effective in prevention. The residual limb is exposed to a new environment when using a prosthesis. New environmental factors including friction, shear forces, sustained pressure, and humidity can all act as contributors to contact dermatitis [32]. Numerous prosthetic materials themselves may be allergens. Determining whether the reaction is localized to the area in contact with the prosthesis or extends to the whole limb aids in identifying the offending agent and allows a change to another liner or prosthesis. Temporizing management would include topical steroid application and prosthetic rest.

\section{Acroangiodermatitis}

Acroangiodermatitis frequently causes pain and pruritus on lower-limb amputation stumps. It is a benign angioproliferative disease resulting from a proliferation of fibroblasts and small vessels that presents as violaceous macules and patches. Acroangiodermatitis has generally been associated with the use of suction socket prostheses in the amputee literature [32] and may be due to improper prosthesis fit. Treatment typically consists of correcting any circulatory disturbance and using rest, elevation, and compression of the lesion and ensuring optimal socket fit and suspension. Other case reports publish treatment options including topical steroids, oral erythromycin, or cryotherapy [46].

\section{Conclusion}

The appropriate management of residual limb complications is time consuming and frequently difficult but may has a substantially positive impact on amputee function and quality of life. The mainstay of therapy for most complications is a trial of non-operative management beginning with a thorough evaluation to determine the cause, followed by conservative measures coupled with prosthetic socket and liner modifications. Operative complications remain unfortunately common, but treatment with surgery does lead to an improved prosthesis utilization and decreased reliance on pain and neuropathic medications [29••]. The importance of individualized therapy in amputee management and the involvement of the multi- 
disciplinary team in formulating a treatment plan cannot be overemphasized. Despite recent progress, more research is clearly indicated to identify and improve best practices of managing and preventing residual limb complications.

Acknowledgments All of the authors are employees of the US Government, and this work was prepared as part of their official duties. As such, there is no copyright to transfer. The views expressed in this article are those of the authors and do not necessarily reflect the official policy or position of the Department of the Navy, Department of the Army, Department of Defense, nor the U.S. Government. We certify that all individuals who qualify as authors have been listed; each has participated in the conception and design of this work, the analysis of data, the writing of the document, and the approval of the submission of this version; that the document represents valid work; that if we used information derived from another source, we obtained all necessary approvals to use it and made appropriate acknowledgements in the document; and that each takes public responsibility for it. Nothing in the presentation implies any Federal/DOD/DON endorsement. None of the authors received financial support for this study.

\section{Compliance with Ethics Guidelines}

Conflict of Interest BA Pascale and BK Potter both declare no conflicts of interest.

Human and Animal Rights and Informed Consent All studies by the authors involving animal and/or human subjects were performed after approval by the appropriate institutional review boards. When required, written informed consent was obtained from all participants.

\section{References}

Papers of particular interest, published recently, have been highlighted as:

- Of importance

-• Of major importance

1. Organization WH, editor Preamble to the Constitution of the World Health Organization. International Health Conference; 1946; New York.

2. Fergason J, Smith D. Socket considerations for the patient with a transtibial amputation. Clin Orthop Relat Res. 1999;361:76-84.

3. Harris AM, Althausen PL, Kellam J, Bosse MJ, Castillo R. Complications following limb-threatening lower extremity trauma. J Orthop Trauma. 2009;23(1):1-6.

4. Tintle SM, Keeling JJ, Shawen SB, Forsberg JA, Potter BK. Traumatic and trauma-related amputations: part I: general principles and lower-extremity amputations. J Bone Joint Surg Am Vol. 2010;92(17):2852-68.

5. Gottschalk F. Transfemoral amputation. Biomechanics and surgery. Clinc Orthop Relat Res. 1999;361:15-22.

6. Gottschalk F, Stills M. The biomechanics of trans-femoral amputation. Prosthet Orthot Int. 1994;18:12-7.

7. Amputations. In: DG Burris PD, DC Elliot, et al, editor. Emergency War Surgery, 3rd US Revision. 3rd US Revision ed. Washington DC: Washington, DC: Department of the Army, Office of The Surgeon General, Borden Institute; 2004.

8. Herscovici D Jr, Sanders RW, Scaduto JM, Infante A, DiPasquale T. Vacuum-assisted wound closure (VAC therapy) for the management of patients with high-energy soft tissue injuries. J Orthop Trauma. 2003;17(10):683-8.

9. Seligson D, Mehta S, Voos K, Henry S, Johnson J. The use of antibiotic-impregnated polymethylmethacrylate beads to prevent the evolution of localized infection. $\mathbf{J}$ Orthop Trauma. 1992;6:401-6.

10. • Barth RE, Vogely H, Hoepelman A, Peters E. 'To bead or not to bead?' Treatment of osteomelitis and prosthetic joint-associated infections with gentamicin bead chains. Int J Antimicrob Agents. 2011;38(5):371-5. This journal article performed a review of current literature and found that no prospective study has proven PMMA beads to be effective in treating orthopaedic infections, but did report several observational studies having achieved infection control. The authors discuss the pros and cons of using the beads, including the limited side-effect profile of the local antibiotic therapy.

11. Smith D, McFarland L, Sangeorzan B, Reiber G, Czerniecki J. Postoperative dressing and a management strategies for transtibial amputations: a critical review. J Rehabil Res Dev. 2003;40(3):213-24.

12. Nawijn S, van der Linde H, Emmelot C, Hofstad JC. Stump management after trans-tibial amputation: a systematic review. Prosthet Orthot Int. 2005;29:13-26.

13. Potter BK, Granville R, Bagg M, Forsberg JA, Hayda R, Keeling $\mathrm{J}$, et al. Special surgical considerations for the combat casualty with limb loss. In: Lenhart MK, editor. Care of the Combat Amputee: Office of the Surgeon General, Department of the Army, USA; 2009. p. 153-90.

14. Hoaglund F, Jergesen H, Wilson L, Lamoreux L, Roberts R. Evaluation of problems and needs of veteran lower-limb amputees in the San Francisco Bay Area during the period 1977-1980. J Rehabil Res Dev. 1983;20(1):57-71.

15. Smith D, Horn P, Malchow D, Boone D, Reiber G, Hansen S Jr. Prosthetic history, prosthetic charges, and functional outcome of the isolated, traumatic below-knee amputee. J Trauma. 1995;38(1):44-7.

16. Gunawardena N, Seneviratne R, Athauda T. Functional outcomes of unilateral lower limb amputee soldiers in two districs of Sri Lanka. Mil Med. 2006;171(4):283-7.

17. Richardson C, Glenn S, Nurmikko T, Horgan M. Incidence of phantom phenomena including phantom limb pain 6 months after major lower limb amputation in patients with peripheral vascular disease. Clin J Pain. 2006;22(4):353-8.

18. Danshaw C. An anesthetic approach to amputation and pain syndromes. Phys Med Rehabil Clin N Am. 2000;11(3):553-7.

19. • Ajibade A, Akinniyi O, Okoye C. Indications and complications of major limb amputations in kano, Nigeria. Ghana Med J. 2013;47(4):185-8. In a review of the indications and complications of amputation in a Nigerian hospital, the authors reported a high complication rate in their retrospective study, with wound infection being the most common. The majority of amputations preformed were from trauma or gangrene due to vascular injury. The study may have been affected by underreporting from the high rate of loss-to-follow-up patients.

20. Potter BK, Burns TC, Lacap AP, Granville RR, Gajewski DA. Heterotopic ossification following traumatic and combat-related amputations. Prevalence, risk factors, and preliminary results of excision. J Bone Joint Surg Am Vol. 2007;89(3):476-86.

21. Partridge C. Influencing factos in surgical wound healing. J Wound Care. 1998;7(7):350-3.

22. Polfer EM, Forsberg JA, Fleming ME, Potter BK. Neurovascular entrapment due to combat-related heterotopic ossification in the lower extremity. J Bone Joint Surg Am Vol. 2013;95(24): e195(1-6).

23. Ahmed A, Bayol M, Ha S. Adventitious bursae in below knee amputees. Am J Phys Med Rehabil. 1994;73(2):124-9. 
24. Stell I. Management of acute burstis: outcome study of a structured approach. J R Soc Med. 1999;92:516-21.

25. Shawen S, Doukas W, Shrout J, Ficke J, Potter BK, Hayda R, et al. General surgical principles for the combat casualty with limb loss. In: Lenhart MK, editor. Care of the Compat Amputee: Office of the Surgeon General, Department of the Army, United States of America; 2009. p. 117-51.

26. Henrot P, Stines J, Walter F, Martinet N, Paysant J, Blum A. Imaging of the painful lower limb stump. Radiographics. 2000;20 Spec No: S219-35.

27. • O'Reilly MA, O'Reilly P, O'Reilly H, Sullivan J, Sheahan J. High-resolution ultrasound findings in the symptomatic residual limbs of amputees. Military Medicine. 2013;178(12):1291-7. High-resolution ultrasound is increasingly being used in the American medical system. In this article, the authors study 133 patients over a 2 year period and use U/S to diagnose a range of pathologies, with neuromas being the most common. They were also able to identify inflamed bursae, thinning of overlying soft tissue, and heterotopic ossification. The authors conclude that ultrasound is a useful modality for assessing symptomatic residual limbs with the added benefit of direct communication with the patient during the exam.

28. Nolan W, Eaton R. Painful neuromas of the upper extremity and postneurectomy pain. In: Omer G, Spinner M, Van Beek A, editors. Management of peripheral nerve problems. Philadelphia: WB Saunders; 1998. p. 146-9.

29. • Tintle SM, Shawen SB, Forsberg JA, Gajewski DA, Keeling JJ, Andersen RC, et al. Reoperation after combat-related major lower extremity amputations. J Orthop Trauma. 2014;28(4):232-7. The authors identify complication rates leading to reoperation of trauma-related amputations, which is not common in the literature. They reported a $53 \%$ reoperation rate out of 300 limbs, commonly with multiple indications for reoperation. The most common indication was postoperative wound infection $(27 \%)$, with symptomatic heterotopic ossification not far behind (24\%). This study confirms the common presence of symptomatic residual limbs and showed that surgical revision leads to improve ambulatory rate and decreased use of neuropathic and narcotic pain medication.

30. Souza JM, Cheesborough JE, Ko JH, Cho MS, Kuiken TA, Dumanian GA. Targeted muscle reinnervation: a novel approach to postamputation neuroma pain. Clin Orthop Relat Res. 2014. doi:10.1007/s11999-014-3528-7.

31. Meulenbelt HE, Geertzen JH, Jonkman MF, Dijkstra PU. Determinants of skin problems of the stump in lower-limb amputees. Arch Phys Med Rehabil. 2009;90(1):74-81.

32. Meulenbelt H, Geertzen JH, Dijkstra PU, Jonkman MF. Skin problems in lower limb amputees: an overview by case reports. JEADV. 2007;21:147-55.

33. Salawu A, Middleton C, Gilberston A, Kodavali K, Neumann V. Stump ulcers and continued prosthtic limb use. Prosthet Orthot Int. 2006;30:279-85.

34. • Traballesi M, DeLussu A, Fusco A, Iosa M, Averna T, Pellegrini R, et al. Residual limb wounds or ulcers heal in transtibial amputees using an active suction socket system. A randomized controlled study. Eur J Phys Rehabil Med. 2012;48:613-23. This prospective study of patients with dysvascular transtibial amputations and open residual limb wounds reported that a vacuumassisted socket system (VASS) allowed for earlier prosthesis fitting and faster rehabilitation of walking capabilities. The VASS prothesis did not increase pain or inhibit wound healing during the study period. This may be due to the change in pressure during the swing and stance phases, increasing circulation. Though, having a small sample size, this was the first longitudinal clinical study to analyze the effects of VASS use in amputees affected by ulcers.

35. Mahaisavariya B, Mahaisavariya P. Marjolin's ulcer complicating a poorly fabricated prosthesis. Injury. 1991;22:423-4.

36. Rush P, Wong J, Kirsh J, Devlin M. Osteopenia in patients with aboe knee amputation. Arch Phys Med Rehabil. 1994;75:12-5.

37. Yazicioglu K, Tugcu I, Yilmaz B, Goktepe AS, Mohur H. Osteoporosis: a factor on residual limb pain in traumatic transtibial amputations. Prosthet Orthot Int. 2008;32(2):172-8.

38. Royer T, Koenig M. Joint loading and bone mineral density in persons with unilateral, trans-tibial amputation. Clin Biomech. 2005;20:1119-25.

39. •• Flint JH, Wade AM, Stocker DJ, Pasquina PF, Howard RS, Potter BK. Bone mineral density loss after combat-related lower extremity amputation. J Ortho Trauma. 2014;28(4):238-44. The authors reported a rate of low bone mineral density (BMD) of $42 \%$, with significant risk factors being prolonged time to first ambulation and more proximal amputation level. Because previous studies indicate that bone loss occurs in amputees, and the military amputee population experiences stress fractures or lowenergy fragility fractures despite a comprehensive rehabilitation program, the authors sought to describe incidence, severity, and associated factors for development of BMD. Their findings support the idea that BMD loss is related to disuse atrophy, and call for prospective studies using serial DEXA scans to quantify reponse to therapetic treatment of low BMD, and response to regular weight bearing status.

40. Tsionos I, Leclercq C, Rochet J. Heterotopic ossification of the elbow in patients with burns. Results after early excision. J Bone Joint Surg Br. 2004;86:396-403.

41. • Matsumoto ME, Khan M, Jayabalan P, Ziebarth J, Munin MC. Heterotopic ossification in civilians with lower limb amputations. Arch Phys Med Rehabil. 2014;95(9):1710-1713. The authors are the first to report a estimate of heterotopic ossification prevalence in a cohort of adult civilian amputees. While military populations are reported to experience rates upto $64 \%$, this retrospective study reports civilian prevalence of $23 \%$, which is still clinically significant. Additionally, no associations between the presence of $\mathrm{HO}$ and the etiology of the amputation or presence of pain was found. The authors also report only 1 patient having developed severe $\mathrm{HO}$, as compared with $26 \%$ in military population.

42. Forsberg JA, Potter BK. Heterotopic Ossification in Wartime Wounds. J Surg Orthop Adv. 2010;19(1):54-61.

43. Haran M, Bhuta T, Lee B. Pharmacological interventions for treating acute heterotopic ossification. Cochrane Database Syst Rev. 2010. doi:10.1002/14651858.CD003321.pub4.

44. Dudek NL, Marks MB, Marshall SC, Chardon JP. Dermatologic conditions associated with use of a lower-extremity prosthesis. Arch Phys Med Rehabil. 2005;86(4):659-63.

45. Kristinsson O. The ICEROSS concept: a discussion of a philosophy. Prosthet Orthot Int. 1993;17:49-55.

46. Ozkaya D, Ozlem S, NOnsun N, Ulusal H, Demirkesen C. Nonhealing ulcer of the foot: early onset unilateral Mali-type acroangiodermatitis. Acta Dermatovenerolica. 2013;22:49-51. 